\title{
Front Matter: Volume 7272
}

, "Front Matter: Volume 7272," Proc. SPIE 7272, Metrology, Inspection, and Process Control for Microlithography XXIII, 727201 (7 April 2009); doi: 10.1117/12.829695

SPIE. Event: SPIE Advanced Lithography, 2009, San Jose, California, United States 


\section{PROCEEDINGS OF SPIE}

\section{Metrology, Inspection, and Process Control for Microlithography XXIII}

John A. Allgair

Christopher J. Raymond

Editors

23-26 February 2009

San Jose, California, United States

Sponsored by

SPIE

Cooperating Organization

SEMATECH Inc. (United States)

Published by

SPIE 
The papers included in this volume were part of the technical conference cited on the cover and title page. Papers were selected and subject to review by the editors and conference program committee. Some conference presentations may not be available for publication. The papers published in these proceedings reflect the work and thoughts of the authors and are published herein as submitted. The publisher is not responsible for the validity of the information or for any outcomes resulting from reliance thereon.

Please use the following format to cite material from this book:

Author(s), "Title of Paper," in Metrology, Inspection, and Process Control for Microlithography XXIII, edited by John A. Allgair, Christopher J. Raymond, Proceedings of SPIE Vol. 7272 (SPIE, Bellingham, WA, 2009) Article CID Number.

ISSN 0277-786X

ISBN 9780819475251

Published by

SPIE

P.O. Box 10, Bellingham, Washington 98227-0010 USA

Telephone +1 3606763290 (Pacific Time) · Fax +1 3606471445

SPIE.org

Copyright (C) 2009, Society of Photo-Optical Instrumentation Engineers

Copying of material in this book for internal or personal use, or for the internal or personal use of specific clients, beyond the fair use provisions granted by the U.S. Copyright Law is authorized by SPIE subject to payment of copying fees. The Transactional Reporting Service base fee for this volume is $\$ 18.00$ per article (or portion thereof), which should be paid directly to the Copyright Clearance Center (CCC), 222 Rosewood Drive, Danvers, MA 01923. Payment may also be made electronically through CCC Online at copyright.com. Other copying for republication, resale, advertising or promotion, or any form of systematic or multiple reproduction of any material in this book is prohibited except with permission in writing from the publisher. The CCC fee code is 0277-786X/09/ $\$ 18.00$.

Printed in the United States of America.

Publication of record for individual papers is online in the SPIE Digital Library.

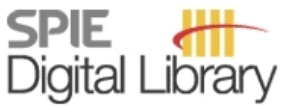

SPIEDigitalLibrary.org

Paper Numbering: Proceedings of SPIE follow an e-First publication model, with papers published first online and then in print and on CD-ROM. Papers are published as they are submitted and meet publication criteria. A unique, consistent, permanent citation identifier (CID) number is assigned to each article at the time of the first publication. Utilization of CIDs allows articles to be fully citable as soon they are published online, and connects the same identifier to all online, print, and electronic versions of the publication. SPIE uses a six-digit CID article numbering system in which:

- The first four digits correspond to the SPIE volume number.

- The last two digits indicate publication order within the volume using a Base 36 numbering system employing both numerals and letters. These two-number sets start with 00, 01, 02, 03, 04, $05,06,07,08,09,0 A, 0 B \ldots 0 Z$, followed by 10-1Z, 20-2Z, etc.

The CID number appears on each page of the manuscript. The complete citation is used on the first page, and an abbreviated version on subsequent pages. Numbers in the index correspond to the last two digits of the six-digit CID number. 


\section{Contents}

\section{Part One}

xix Symposium Committees

xxi Conference Committee

SESSION 1 KEYNOTE SESSION

727202 Improving optical measurement accuracy using multi-technique nested uncertainties (Keynote Paper) [7272-01]

R. M. Silver, N. F. Zhang, B. M. Barnes, H. Zhou, A. Heckert, R. Dixson, T. A. Germer, National Institute of Standards and Technology (United States); B. Bunday, SEMATECH, Inc. (United States)

727203 The measurement uncertainty challenge of advanced patterning development (Keynote Paper) [7272-02]

N. Rana, C. Archie, W. Lu, B. Banke, IBM Corp. (United States)

\section{SESSION 2 METHODS FOR TODAY}

727204 CD-SEM parameter influence on image resolution and measurement accuracy [7272-03] B. Bunday, SEMATECH, Inc. (United States); U. Kramer, Qimonda Dresden GmbH \& Co. OHG (Germany)

727205 Role of CDAFM in achieving accurate OPC modeling [7272-04]

V. A. Ukraintsev, Veeco Instruments Inc. (United States)

727206 Sampling for advanced overlay process control [7272-05]

C. Kato, H. Kurita, KLA-Tencor Japan Co. Ltd. (Japan); P. Izikson, KLA-Tencor (Israel);

J. C. Robinson, KLA-Tencor Corp. (United States)

727207 Simultaneous overlay and CD measurement for double patterning: scatterometry and RCWA approach [7272-06]

J. Li, Z. Liu, S. Rabello, P. Dasari, Nanometrics Inc. (United States); O. Kritsun, C. Volkman, GLOBALFOUNDRIES Inc. (United States); J. Park, L. Singh, Spansion Inc. (United States)

727209 Reference metrology in a research fab: the NIST clean calibrations thrust [7272-08]

R. Dixson, J. Fu, N. Orji, T. Renegar, A. Zheng, T. Vorburger, A. Hilton, M. Cangemi, L. Chen, M. Hernandez, R. Hajdaj, National Institute of Standards and Technology (United States);

M. Bishop, A. Cordes, SEMATECH, Inc. (United States) 
7272 OA Evaluation of a new metrology technique to support the needs of accuracy, precision, speed, and sophistication in near-future lithography [7272-09]

C.-M. Ke, J. Hu, W. Wang, J. Huang, H. L. Chung, C. R. Liang, V. Shih, H. H. Liu, H. J. Lee, J. Lin, Y. D. Fan, Taiwan Semiconductor Manufacturing Co. Ltd. (Taiwan); K. Bhattacharyya, M. van der Schaar, N. Wright, K. Yuan, V. Wang, C. Wang, S. Lin, M. Shahrjerdy, A. Fuchs, K. van der Mast, ASML Netherlands B.V. (Netherlands)

7272 OB MOSAIC: a new wavefront metrology [7272-10]

C. N. Anderson, Univ. of California, Berkeley (United States); P. P. Naulleau, Lawrence Berkeley National Lab. (United States)

7272 OD Immersion specific error contribution to overlay control [7272-12]

K. D'havé, D. Laidler, S. Cheng, IMEC (Belgium)

7272 OE Overlay similarity: a new overlay index for metrology tool and scanner overlay fingerprint methodology [7272-13]

C.-M. Ke, C.-P. Kao, Y. Wang, J. Hu, C.-Y. Chang, Y.-J. Tsai, A. Yen, B. J. Lin, Taiwan

Semiconductor Manufacturing Co. Ltd. (Taiwan)

7272 OF Tabletop coherent diffractive microscopy with extreme ultraviolet light from high harmonic generation (Best Student Paper Award) [7272-14]

D. A. Raymondson, R. L. Sandberg, Univ. of Colorado at Boulder (United States) and National Institute of Standards and Technology (United States); W. F. Schlotter, Univ. Hamburg (Germany); K. S. Raines, Univ. of California, Los Angeles (United States); C. La-o-Vorakiat, E. Townsend, Univ. of Colorado at Boulder (United States) and National Institute of Standards and Technology (United States); A. Sakdinawat, Lawrence Berkeley National Lab. (United States); A. Paul, Univ. of Colorado at Boulder (United States) and National Institute of Standards and Technology (United States); J. Miao, Univ. of California, Los Angeles (United States); M. M. Murnane, H. C. Kapteyn, Univ. of Colorado at Boulder (United States) and National Institute of Standards and Technology (United States)

\section{SESSION 4 OVERLAY}

$72720 \mathrm{O}$ Overlay metrology for double patterning processes [7272-15]

P. Leray, S. Cheng, D. Laidler, IMEC (Belgium); D. Kandel, M. Adel, B. Dinu, M. Polli, KLA-Tencor Corp. (Israel); M. Vasconi, STMicroelectronics (Italy); B. Salski, QWED (Poland)

$7272 \mathrm{OH} \quad$ Implementation of the high order overlay control for mass production of 40nm node logic devices [7272-18]

D. Umeda, M. Miyasaka, T. Uchiyama, NEC Electronics Corp. (Japan)

7272 Ol Using intrafield high-order correction to achieve overlay requirement beyond sub-40nm node [7272-20]

C. Y. Huang, C. F. Chue, A.-H. Liu, W. B. Wu, C. L. Shih, Nanya Technology Corp. (Taiwan); T.-B. Chiou, ASML Technology Development Ctr. (Taiwan); J. Lee, O. Chen, ASML Center of Excellence (ACE) (Taiwan); A. Chen, ASML Technology Development Ctr. (Taiwan) 
7272 0J Polar Correction: new overlay control method for higher-order intra-field error dependent on the wafer coordinates [7272-21]

M. Takakuwa, K. Toriumi, N. Komine, K. Ishigo, T. Kono, T. Nakasugi, T. Higashiki, Toshiba Corp. (Japan)

7272 OK Effects of plasma spatial profile on conversion efficiency of laser produced plasma sources for EUV lithography [7272-73]

A. Hassanein, V. Sizyuk, T. Sizyuk, S. Harilal, Purdue Univ. (United States)

\section{SESSION $5 \quad$ LINE EDGE/WIDTH ROUGHNESS}

7272 OL Dark-field optical scatterometry for line width roughness metrology [7272-22]

G. V. Zhuang, S. Spielman, J. Fielden, D. C. Wack, L. Poslavsky, KLA-Tencor Corp. (United States); B. D. Bunday, SEMATECH, Inc. (United States)

7272 OM A CD AFM study of the plasma impact on 193nm photoresist LWR: role of plasma UV and ions [7272-23]

E. Pargon, M. Martin, K. Menguelti, L. Azarnouche, UJF/INP, LTM-CNRS Grenoble (France);

J. Foucher, CEA-LETI Minatec (France); O. Joubert, UJF/INP, LTM-CNRS Grenoble (France)

7272 ON SEM metrology damage in polysilicon line and its impact on LWR evaluation [7272-24] S.-B. Wang, W.-Y. Lee, Y. H. Chiu, H. J. Tao, Y. J. Mii, Taiwan Semiconductor Manufacturing Co. Ltd. (Taiwan)

$727200 \quad$ Process variation monitoring (PVM) by wafer inspection tool as a complementary method to CD-SEM for mapping LER and defect density on production wafers [7272-25]

S. Shabtay, Y. Blumberg, S. Levi, G. Greenberg, D. Harel, A. Conley, D. Meshulach, K. Kan, I. Dolev, S. Kumar, K. Mendel, Applied Materials (Israel); K. Goto, N. Yamaguchi, Applied Materials, Inc. (Japan); Y. Iriuchijima, S. Nakamura, S. Nagaoka, T. Sekito, Nikon Corp. (Japan)

\section{SESSION 6 SEM I}

$72720 Q \quad$ Validation of CD-SEM etching residue evaluation technique for MuGFET structures [7272-27] M. Isawa, M. Tanaka, T. Maeda, K. Watanabe, Hitachi High-Technologies Corp. (Japan); T. Vandeweyer, N. Collaert, R. Rooyackers, IMEC (Belgium)

7272 OR Sensitivity of SEM width measurements to model assumptions [7272-28] J. S. Villarrubia, National Institute of Standards and Technology (United States); Z. J. Ding, Univ. of Science and Technology of China (China)

7272 OS Accurate electrical prediction of memory array through SEM-based edge-contour extraction using SPICE simulation [7272-29]

E. Shauly, I. Rotstein, Tower Semiconductor Ltd. (Israel); R. Peltinov, S. Latinski, O. Adan, S. Levi, O. Menadeva, Applied Materials, Inc. (Israel) 
7272 OT Developing an uncertainty analysis for optical scatterometry [7272-30]

T. A. Germer, National Institute of Standards and Technology (United States); H. J. Patrick, National Institute of Standards and Technology (United States) and KT Consulting, Inc. (United States); R. M. Silver, National Institute of Standards and Technology (United States); B. Bunday, SEMATECH, Inc. (United States)

7272 OU Effect of line-width roughness on optical scatterometry measurements [7272-31] B. C. Bergner, National Institute of Standards and Technology (United States), The Univ. of North Carolina, Charlotte (United States), and KT Consulting, Inc. (United States); T. A. Germer, National Institute of Standards and Technology (United States); T. J. Suleski, The Univ. of North Carolina, Charlotte (United States)

7272 OV Product-driven material characterization for improved scatterometry time-to-solution [7272-32]

A. Vaid, Advanced Micro Devices, Inc. (United States); C. Hartig, AMD LLC \& Co. KG (Germany); M. Sendelbach, IBM Corp. (United States); C. Bozdog, H. K. Kim, M. Sendler, Nova Measuring Instruments, Inc. (United States); Y. Cohen, V. Kucherov, B. Brill, S. Stepanov, Nova Measuring Instruments, Ltd. (Israel)

$7272 \mathrm{OW}$ Manufacturing implementation of scatterometry and other techniques for $\mathbf{3 0 0}-\mathbf{m m}$ lithography tool controls [7272-33]

T. Wiltshire, D. Corliss, T. Brunner, C. Ausschnitt, R. Young, R. Nielson, E. Hwang, J. lannucci, Jr., IBM Corp. (United States)

\section{SESSION 8 SEM II}

7272 OY Methodologies for evaluating CD-matching of CD-SEM [7272-36]

H. Kawada, Hitachi High-Technologies Corp. (Japan); C.-M. Ke, Y.-C. Cheng, Y.-H. Wang, Taiwan Semiconductor Manufacturing Co. Ltd. (Taiwan)

$72720 Z$ Calibration of a scanning electron microscope in the wide range of magnifications for the microscope operation in the integrated circuit production line [7272-37] V. P. Gavrilenko, Ctr. for Surface and Vacuum Research (Russian Federation); Yu. A. Novikov, A. V. Rakov, A.M. Prokhorov General Physics Institute (Russian Federation); P. A. Todua, Ctr. for Surface and Vacuum Research (Russian Federation); Ch. P. Volk, Mikron Corp. (Russian Federation)

727210 CD-SEM tool stability and tool-to-tool matching management using image sharpness monitor [7272-38]

H. Abe, Y. Ishibashi, Y. Yamazaki, Toshiba Corp. (Japan); A. Kono, T. Maeda, A. Miura, S. Koshihara, D. Hibino, Hitachi High-Technologies Corp. (Japan)

727211 Performance verification of resist loss measurement method using top-view CD-SEM images for hyper-NA lithography [7272-39]

M. Osaki, Hitachi, Ltd. (Japan); M. Tanaka, Hitachi High-Technologies Corp. (Japan); C. Shishido, Hitachi, Ltd. (Japan); S. Cheng, D. Laidler, M. Ercken, E. Altamirano, IMEC (Belgium) 
727212 Diffraction-based overlay metrology for double patterning technologies [7272-41]

P. Dasari, R. Korlahalli, J. Li, N. Smith, Nanometrics Inc. (United States); O. Kritsun, C. Volkman, GLOBALFOUNDRIES Inc. (United States)

727214 Through-focus scanning and scatterfield optical methods for advanced overlay target analysis [7272-43]

R. Attota, M. Stocker, R. Silver, A. Heckert, National Institute of Standards and Technology (United States); H. Zhou, KT Consulting Inc. (United States); R. Kasica, L. Chen, R. Dixson, G. Orji, B. Barnes, National Institute of Standards and Technology (United States);

P. Lipscomb, SEMATECH, Inc. (United States)

\section{SESSION 10 MASK METROLOGY}

727215 Cr migration on 193nm binary photomasks [7272-44]

J. Bruley, IBM Thomas J. Watson Research Ctr. (United States); G. Burr, IBM Almaden Research Ctr. (United States); R. E. Davis, P. Flaitz, IBM Corp. (United States); W. D. Hinsberg, F. A. Houle, D. C. Miller, IBM Almaden Research Ctr. (United States); M. Pike, J. Rankin, IBM Corp. (United States); A. Wagner, IBM Thomas J. Watson Research Ctr. (United States); A. Watts, IBM Corp. (United States)

727216 Compute resource management and TAT control in mask data prep [7272-46] A. Nouh, K. Jantzen, M. Park, H. T. Vu, Mentor Graphics Corp. (United States)

727217 Investigation of phase distribution using Phame in-die phase measurements [7272-47] U. Buttgereit, S. Perlitz, Carl Zeiss SMS GmbH (Germany)

727218 Image library approach to evaluating parametric uncertainty in metrology of isolated feature width [7272-48]

J. Potzick, National Institute of Standards and Technology (United States)

\section{SESSION 11 INSPECTION}

727219 New inspection technology for hole pattern by Fourier space on hp $4 \mathrm{x}-\mathrm{nm}$ generation [7272-49]

A. Kawai, F. Hayano, K. Endo, Nikon Corp. (Japan); K. Yoshino, Y. Yamazaki, Toshiba Corp. (Japan)

72721 A Development of optical simulation tool for defect inspection [7272-50]

T. Fujii, Y. Konno, N. Okada, K. Yoshino, Y. Yamazaki, Toshiba Corp. (Japan)

7272 1B Phenomenology of electron-beam-induced photoresist shrinkage trends [7272-138] B. Bunday, A. Cordes, J. Allgair, SEMATECH, Inc. (United States); V. Tileli, Univ. at Albany, SUNY (United States); Y. Avitan, R. Peltinov, M. Bar-zvi, O. Adan, Applied Materials (Israel); E. Cottrell, S. Hand, Veeco Metrology Inc. (United States) 
7272 1C Systematic defect filtering and data analysis methodology for design based metrology [7272-53]

H. Yang, J. Kim, T. Lee, A. Jung, G. Yoo, D. Yim, S. Park, Hynix Semiconductor Inc. (Korea, Republic of); T. Hasebe, M. Yamamoto, NanoGeometry Research, Inc. (Japan); J. Cai, Anchor Semiconductor, Inc. (United States)

7272 1D Quantitative measurement of voltage contrast in SEM images for in-line resistance inspection of wafers manufactured for SRAM [7272-54]

M. Matsui, T. Odaka, Hitachi, Ltd. (Japan); H. Nagaishi, K. Sakurai, Renesas Technology Corp. (Japan)

7272 IE Study of device leakage of $45 \mathrm{~nm}$ node with different SRAM layouts using an advanced e-beam inspection system [7272-55]

H. Xiao, L. Ma, Y. Zhao, J. Jau, Hermes Microvision, Inc. (United States)

\section{SESSION 12 PROCESS CONTROL}

7272 IF Hotspot monitoring system with contour-based metrology [7272-56]

A. Kawamoto, Y. Tanaka, S. Tsuda, K. Shibayama, S. Furukawa, H. Abe, T. Mitsui, Y. Yamazaki, Toshiba Corp. (Japan)

$72721 \mathrm{G}$ Outlier detection by fuzzy classification method for model building [7272-57]

M. K. Top, STMicroelectronics (France) and LTM-CNRS (France); Y. Troviller, CEA-Leti (France); V. Farys, STMicroelectronics (France); D. Fuard, LTM-CNRS (France); E. Yesilada, C. Martinelli, M. Said, F. Foussadier, STMicroelectronics (France); P. Schiavone, LTM-CNRS (France) and CNRS, Georgia Institute of Technology (United States)

$72721 \mathrm{H} \quad$ Monitoring measurement tools: new methods for driving continuous improvements in fleet measurement uncertainty [7272-58]

E. Solecky, C. Archie, M. Sendelbach, R. Fiege, M. Zaitz, D. Shneyder, C. Strocchia-rivera, A. Munoz, S. Rangarajan, W. Muth, A. Brendler, B. Banke, IBM Corp. (United States); B. Schulz, C. Hartig, J.-T. Hoeft, AMD Fab36 LLC \& Co. KG (Germany); A. Vaid, M. Kelling, AMD, Inc. (United States); B. Bunday, J. Allgair, SEMATECH, Inc. (United States)

$727211 \quad$ Two-dimensional dose and focus-error measurement technology for exposure tool management in half-pitch $3 x$ generation [7272-59]

K. Fukazawa, Y. Kudo, Y. Fujimori, Nikon Corp. (Japan); K. Yoshino, Y. Yamazaki, Toshiba Corp. (Japan)

7272 1J Increased uniformity control in a 45nm polysilicon gate etch process [7272-124] B. Parkinson, D. Prager, M. Funk, R. Sundararajan, Tokyo Electron America, Inc. (United States); A. Yamashita, Tokyo Electron AT Ltd. (Japan); K. Bandy, E. Meyette, IBM Corp. (United States)

$72721 \mathrm{~K}$ The measurement uncertainty challenge for the future technological nodes production and development [7272-125]

J. Foucher, P. Faurie, A.-L. Foucher, M. Cordeau, CEA, LETI, Minatec (France); V. Farys, STMicroelectronics (France) 
7272 1L Angle-resolved scatterfield microscope for linewidth measurement [7272-63]

D.-M. Shyu, Y.-S. Ku, W.-T. Hsu, Industrial Technology Research Institute (Taiwan)

$72721 \mathrm{M}$ Optical CD metrology model evaluation and refining for manufacturing [7272-64]

S.-B. Wang, C. L. Huang, Y. H. Chiu, H. J. Tao, Y. J. Mii, Taiwan Semiconductor Manufacturing Co. Ltd. (Taiwan)

$72721 \mathrm{~N} \quad$ Uncertainty and sensitivity analysis and its applications in OCD measurements [7272-65]

P. Vagos, J. Hu, Z. Liu, S. Rabello, Nanometrics Inc. (United States)

\section{SESSION 14 REFERENCE METROLOGY}

7272 IP AFM method for sidewall measurement through CNT probe deformation correction and its accuracy evaluation [7272-70]

M. Watanabe, S. Baba, T. Nakata, Hitachi, Ltd. (Japan); H. Itoh, National Institute of Advanced Industrial Science and Technology (Japan); T. Morimoto, S. Sekino, Hitachi Construction Machinery Co., Ltd. (Japan)

\section{POSTER SESSION}

7272 1Q Alignment method of self-aligned double patterning process [7272-16] M.-F. Tsai, J.-C. Lai, Y.-S. Chang, C.-C. Lin, Powerchip Semiconductor Corp. (Taiwan)

7272 IR Application results of lot-to-lot high-order overlay correction for sub-60-nm memory device fabrication [7272-19]

J. Shin, S. Nam, T. Kim, Y.-K. Bae, J. Lee, SAMSUNG Electronics Co., Ltd. (Korea, Republic of)

7272 is Fast mask CD uniformity measurement using zero order diffraction from memory array pattern [7272-45]

J. Heo, J. Park, J. Yeo, S. Choi, W. Han, SAMSUNG Electronics Co., Ltd. (Korea, Republic of)

7272 1T Requirements of the inspection for double patterning technology reticles [7272-51]

W. Cho, W.-S. Kim, S.-J. Sohn, S. Lee, J. Choi, Y. Kim, H. Cho, SAMSUNG Electronics Co., Ltd. (Korea, Republic of)

$72721 \mathrm{U}$ Sensitivity improvement and noise reduction of array CD mapping on memory device using inspection tool [7272-52]

J.-H. Yeo, B.-O. Cho, J.-H. Park, J. Hur, S.-H. Woo, S. Choi, C.-H. Park, SAMSUNG Electronics

Co., Ltd. (Korea, Republic of)

$72721 \mathrm{~V} \quad$ Analysis of systematic errors in lateral shearing interferometry for EUV optical testing [7272-71]

R. Miyakawa, Univ. of California, Berkeley (United States) and Lawrence Berkeley National Lab. (United States); P. Naulleau, K. Goldberg, Lawrence Berkeley National Lab. (United States)

7272 1X Haze generation model and prevention techniques for sulfate free cleaned mask [7272-74] M. Patil, J.-M. Kim, I.-B. Hur, S.-S. Choi, PKL-Photronics Co., Ltd. (Korea, Republic of) 
$72721 \mathrm{R}$ Resist-based polarization monitoring with phase-shift masks at 1.35 numerical aperture [7272-76]

G. McIntyre, IBM Corp. (United States); R. Tu, Benchmark Technologies (United States)

727220 An investigation of perfluoroalkylamine contamination control [7272-79]

A. J. Dallas, D. Zastera, Donaldson Co., Inc. (United States)

727221 Sub-nanometer broadband measurement of elastic displacements in optical metrology frames and other critical elements [7272-80]

G. Kessenich, S. Bhola, B. Pletner, W. Horth, IPTRADE, Inc. (United States); A. Hosoi, Massachusetts Institute of Technology (United States)

\section{Part Two}

727222 Measurement of low molecular weight silicon AMC to protect UV optics in photolithography environments [7272-81]

J. M. Lobert, C. M. Miller, A. Grayfer, A. M. Tivin, Entegris, Inc. (United States)

727223 Positive identification of lithographic photoresists using real-time index of refraction monitoring for reduced cost of ownership [7272-82]

R. Jee, S. Pepper, D. Stedman, Swagelok Co. (United States)

727224 Sub-50-nm pitch size grating reference for CD-SEM magnification calibration [7272-84] Y. Nakayama, J. Yamamoto, Hitachi, Ltd. (Japan); H. Kawada, Hitachi High-Technologies Corp. (Japan)

727226 Effective purging solution to reticle haze formation [7272-86]

W.-J. Tseng, S.-H. Chiou, Rexchip Electronics Corp. (Taiwan); M.-C. Chiu, P.-S. Lee, Gudeng Precision Industrial Co., Ltd. (Taiwan)

727227 Measurement of dimensions of resist mask elements below $100 \mathrm{~nm}$ with help of a scanning electron microscope [7272-87]

V. P. Gavrilenko, Ctr. for Surface and Vacuum Research (Russian Federation); V. A. Kalnov, Institute of Physics and Technology (Russian Federation); Yu. A. Novikov, A.M. Prokhorov General Physics Institute (Russian Federation); A. A. Orlikovsky, Institute of Physics and Technology (Russian Federation); A. V. Rakov, A.M. Prokhorov General Physics Institute (Russian Federation); P. A. Todua, Ctr. for Surface and Vacuum Research (Russian Federation); K. A. Valiev, E. N. Zhikharev, Institute of Physics and Technology (Russian Federation)

727228 Aerial imaging for FABs: productivity and yield aspects [7272-88] I. Englard, Applied Materials BV (Netherlands); Y. Cohen, Y. Elblinger, S. Attal, N. Berns, L. Shoval, M. Ben-Yishai, S. Mangan, Applied Materials (Israel)

727229 Improved mask-based CD uniformity for gridded-design-rule lithography [7272-89] L. Faivishevsky, S. Khristo, A. Sagiv, S. Mangan, Applied Materials (Israel) 
7272 2A Study of advanced mask inspection optics with super-resolution method for nextgeneration mask fabrication [7272-90]

R. Hirano, M. Hirono, R. Ogawa, N. Kikuiri, Advanced Mask Inspection Technology, Inc. (Japan); K. Takahara, H. Hashimoto, NuFlare Technology, Inc. (Japan); H. Shigemura, Semiconductor Leading Edge Technologies, Inc. (Japan)

7272 2B Novel lithography approach using feed-forward mask-based wafer CDU correction to increase fab productivity and yield [7272-91]

S. Mangan, Applied Materials, Inc. (Israel); E. Byers, Micron Technology, Inc. (United States); D. Rost, MP Mask Technology Ctr., LLC (United States); M. Garrett, M. Carlson, C. Hickman, Micron Technology, Inc. (United States); J. Finders, P. Luehrmann, R. Kazinczi,

I. Minnaert-Janssen, F. Duray, B. Wisse, N. Schoumans, L. Reijnen, T. Theeuwes, ASML Netherlands B.V. (Netherlands); M. Ben-Yishai, Applied Materials, Inc. (Israel); R. Ren, R. Gibson, Applied Materials, Inc. (United States); L. Shoval, Y. Cohen, Y. Elblinger, Applied Materials, Inc. (Israel); I. Englard, Applied Materials B.V. (Netherlands)

$72722 \mathrm{C}$ CD bias reduction in CD-SEM line-width measurement for the $32 \mathrm{~nm}$ node and beyond using the model-based library method [7272-93]

C. Shishido, Hitachi, Ltd. (Japan); M. Tanaka, Hitachi High-Technologies Corp. (Japan); M. Osaki, Hitachi, Ltd. (Japan)

7272 2D New approach for mask-wafer measurement by design-based metrology integration system [7272-94]

T. Maeda, Hitachi High-Technologies Corp. (Japan); K. Hayano, S. Kawashima, H. Mohri, Dai Nippon Printing Co., Ltd. (Japan); H. Sakai, H. Sato, R. Matsuoka, M. Nishihara, S. Sukegawa, Hitachi High-Technologies Corp. (Japan)

$72722 \mathrm{E} \quad$ A practical application of multiple parameters profile characterization (MPPC) using CD-SEM on production wafers using hyper-NA lithography [7272-95]

T. Ishimoto, Hitachi High-Technologies Corp. (Japan); K. Sekiguchi, Hitachi HighTechnologies Europe GmbH (Germany); N. Hasegawa, K. Watanabe, Hitachi HighTechnologies Corp. (Japan); D. Laidler, S. Cheng, IMEC (Belgium)

$72722 \mathrm{~F}$ Improving capability of recipe management on CD-SEM using recipe diagnostic tool [7272-96]

K. Nishiuchi, Panasonic Corp. (Japan); S. Nakano, M. Nishino, Panasonic Semiconductor Engineering Co.,Ltd. (Japan); K. Yang, J. Kakuta, Y. Nakata, S. Koshihara, Hitachi High-Technologies Corp. (Japan)

7272 2G Three-dimensional profile extraction from CD-SEM top-view image [7272-97]

A. Yamaguchi, Y. Momonoi, Hitachi, Ltd. (Japan); K. Murayama, Hitachi Kenki Fine Tech Co., Ltd. (Japan); H. Kawada, Hitachi High-Technologies Corp. (Japan); J. Tanaka, Hitachi, Ltd. (Japan)

7272 2J 3D-AFM booster for mass-production nanoimprint lithography [7272-101] A.-L. Foucher, J. Foucher, S. Landis, CEA-LETI, Minatec (France)

$72722 \mathrm{~L}$ Simulation of secondary electron emission in helium ion microscope for overcut and undercut line-edge patterns [7272-103]

T. Yamanaka, K. Inai, K. Ohya, Univ. of Tokushima (Japan); T. Ishitani, Hitachi High-

Technologies Corp. (Japan) 
7272 2M Nonplanar high-k dielectrics thickness measurement using CD-SAXS [7272-104]

C. Wang, National Institute of Standards and Technology (United States); K.-W. Choi, Intel Corp. (United States); Y.-C. Chen, National Institute of Standards and Technology (United States); J. Price, SEMATECH, Inc. (United States); D. L. Ho, R. L. Jones, C. Soles, E. K. Lin, W. Wu, National Institute of Standards and Technology (United States); B. D. Bunday, SEMATECH, Inc. (United States)

7272 2N High-precision CD matching monitoring technology using profile gradient method for the 32-nm technology generation [7272-105]

T. Ikegami, A. Kono, T. Maeda, Hitachi High-Technologies Corp. (Japan); M. Osaki,

C. Shishido, Hitachi Ltd. (Japan)

727220 CD budget analysis on sub-50nm DRAM device: global CD variation to local CD variation [7272-106]

C. Hwang, J. Park, J. Yeo, S.-W. Choi, C.-H. Park, SAMSUNG Electronics Co., Ltd. (Korea, Republic of)

7272 2P Intrafield process control for 45 nm CMOS logic patterning [7272-107]

B. Le Gratiet, J. Massin, A. Ostrovski, C. Monget, M. Decaux, N. Thivolle, R. Faure, F. Baron, J.-D. Chapon, K. Dabertrand, F. Sundermann, P. Gouraud, L. Babaud, L. Thevenon, N. Cluet, B. VandeWalle, STMicroelectronics (France)

$72722 \mathrm{C} \quad$ Contour quality assessment for OPC model calibration [7272-108]

P. Filitchkin, T. Do, I. Kusnadi, J. L. Sturtevant, Mentor Graphics Corp. (United States);

P. de Bisschop, J. Van de Kerkhove, IMEC (Belgium)

$72722 \mathrm{R} \quad$ Applications of AFM in semiconductor R\&D and manufacturing at $\mathbf{4 5} \mathrm{nm}$ technology node and beyond [7272-109]

M.-K. Lee, Veeco Instruments Inc. (United States); M. Shin, SAMSUNG Electronics Co., Ltd. (Korea, Republic of); T. Bao, Veeco Instruments Inc. (United States); C.-G. Song, SAMSUNG Electronics Co., Ltd. (Korea, Republic of); D. Dawson, Veeco Instruments Inc. (United States); D.-C. Ihm, SAMSUNG Electronics Co., Ltd. (Korea, Republic of); V. Ukraintsev, Veeco Instruments Inc. (United States)

7272 2T WLCD: a new system for wafer level CD metrology on photomasks [7272-111] S. Martin, H. Seitz, W. Degel, U. Buttgereit, T. Scherübl, Carl Zeiss SMS GmbH (Germany)

$72722 \mathrm{U}$ 3D touch trigger probe based on fiber Bragg gratings [7272-112]

B. Ding, Y. Fei, Z. Fan, Hefei Univ. of Technology (China)

7272 2W In-die registration metrology on future-generation reticles [7272-114]

K.-D. Roeth, F. Laske, KLA-Tencor (Germany); H. Kinoshita, D. Kenmochi, HOYA Corp. (Japan); K.-H. Schmidt, D. Adam, KLA-Tencor (Germany)

$72722 \mathrm{X} \quad$ Advanced modeling strategies to improve overlay control for 32-nm lithography processes [7272-115]

A. Minvielle, L. Singh, J. Schefske, J. Reiss, E. Kent, T. Manchester, Spansion Inc. (United States); B. Eichelberger, K. O'Brien, J. Manka, J. C. Robinson, D. Tien, KLA-Tencor Corp. (United States) 
$72722 Y \quad$ Overlay mark optimization using the KTD signal simulation system [7272-117]

A. Marchelli, KLA-Tencor Corp. (Israel); K. Gutjahr, M. Kubis, Qimonda Dresden GmbH \& Co. OHG (Germany); C. Sparka, M. Ghinovker, KLA-Tencor Corp. (Israel); A. Navarra, Qimonda Dresden GmbH \& Co. OHG (Germany); A. Widmann, KLA-Tencor Corp. (Israel)

$72722 Z$ Fast analysis and diagnostics for improving overlay control: moving beyond the black box approach [7272-119]

Y.-A. Liu, W.-M. Wu, H.-C. Lin, J.-C. Lai, Powerchip Semiconductor Corp. (Taiwan);

C.-C. Huang, H.-C. Wu, H. Huang, D. Tien, KLA-Tencor Corp. (United States)

727230 The study and simulation of high-order overlay control including field-by-field methodologies [7272-120]

D. Choi, KLA-Tencor Korea (Korea, Republic of); C. Lee, C. Bang, M. Kim, H. Kang, Hynix Semiconductor Inc. (Korea, Republic of); J. Manka, KLA-Tencor Corp. (United States); S. Yoon, D. Lee, KLA-Tencor Korea (Korea, Republic of); J. C. Robinson, KLA-Tencor Corp. (United States)

727231 Sampling strategy: optimization and correction for high-order overlay control for $45 \mathrm{~nm}$ process node [7272-121]

B. Y. Hsueh, G. K. C. Huang, C.-C. Yu, United Microelectronics Corp. (Taiwan); C.-C. Huang, C.-J. Huang, J. R. Manka, D. Tien, KLA-Tencor Corp. (United States)

727232 Automated overlay recipe setup in high-volume manufacturing: improving performance, efficiency, and robustness [7272-122]

C. Sparka, A. Golotsvan, Y. Avrahamov, W. Sitzmann, D. Tien, KLA-Tencor Corp. (United States)

727233 Optimization of alignment strategy for metal layer on local interconnect integration [7272-123]

J.-K. Ahn, J.-H. Ha, H.-I. Kim, J.-L. Park, J.-S. Choi, T.-J. Lee, MagnaChip Semiconductor, Ltd. (Korea, Republic of)

727234 Challenges of long-term process stability and solutions for better control [7272-126] J. Choi, SAMSUNG Electronics Co., Ltd. (Korea, Republic of); N. Seong, Cymer, Inc. (United States); S. Lee, Y. Kang, SAMSUNG Electronics Co., Ltd. (Korea, Republic of)

727235 Use of 3D metrology for process control [7272-127] B. Rijpers, J. Finders, ASML Netherlands B.V. (Netherlands); H. Suzuki, T. Fujii, SII NanoTechnology Inc. (Japan); Y. Yamazaki, H. Abe, Toshiba Corp. (Japan); F. Pérez-Willard, Carl Zeiss GmbH (Germany)

727236 Track optimization and control for 32nm node double patterning and beyond [7272-128] D. Laidler, IMEC (Belgium); C. Rosslee, SOKUDO Co., Ltd. (United States); K. D'havé, P. Leray, IMEC (Belgium); L. Tedeschi, SOKUDO Co., Ltd. (United States)

727237 Contact area as the intuitive definition of contact CD based on aerial image analysis [7272-129]

N. Polonsky, A. Sagiv, S. Mangan, Applied Materials (Israel)

727238 Focus and dose control for high-volume manufacturing of semiconductor [7272-130]

K. Sentoku, T. Ebihara, H. Ina, Canon Inc. (Japan) 
727239 Efficient use of design-based binning methodology in a DRAM fab [7272-131]

L. Karsenti, Applied Materials (Israel); A. Wehner, A. Fischer, U. Seifert, J. Goeckeritz,

Qimonda Dresden GmbH \& Co. OHG (Germany); M. Geshel, D. Gscheidlen, A. Bartov, Applied Materials (Israel)

7272 3A Proximity matching for ArF and KrF scanners [7272-132]

Y. K. Kim, L. Pohling, N. T. Hwee, J. S. Kim, P. Benyon, Chartered Semiconductor

Manufacturing Ltd. (Singapore); J. Depre, J. Hong, A. Serebriakov, ASML Netherlands B.V. (Netherlands)

7272 3B Scanner matching optimization [7272-133]

M. Kupers, P. Klingbeil, J. Tschischgale, S. Buhl, F. Hempel, Qimonda Dresden GmbH \& Co. OHG (Germany)

7272 3D Comparative study of process window identification methods for $\mathbf{4 5} \mathbf{n m}$ device and beyond [7272-135]

H. Kang, S. Lee, M. Kim, K. Kim, Y. Jeong, SAMSUNG Electronics Co., Ltd. (Korea, Republic of);

Y. Pae, C. Lee, KLA-Tencor Corp. (United States)

$72723 \mathrm{E}$ Improve scanner matching using automated real-time feedback control via scanner match maker (SMM) [7272-136]

S.-H. Chiu, S.-H. Yu, Rexchip Electronics Corp. (Taiwan); M.-H. Tung, Rexchip Electronics Corp. (Taiwan) and Chung-Hua Univ. (Taiwan); L.-K. WU, Y.-T. Yeh, Rexchip Electronics Corp.

(Taiwan); J. Manka, C.-T. Huang, J. Robinson, C.-C. Huang, D. Tien, KLA-Tencor Corp. (United States); Y. Chen, Nikon Precision Tawian Ltd. (Taiwan); K. Makino, Nikon Corp. (Japan); J.-M. Lin, Chung-Hua Univ. (Taiwan)

$72723 \mathrm{H} \quad$ Hole inspection technology using Fourier imaging method [7272-140]

K. Yoshino, K. Tsuchiya, Y. Yamazaki, M. Oote, K. Shibayama, Toshiba Corp. (Japan); A. Kawai, Nikon Corp. (Japan); K. Endo, Toshiba Corp. (Japan)

$727231 \quad$ Investigation of factors causing difference between simulation and real SEM image [7272-143]

M. Kadowaki, A. Hamaguchi, H. Abe, Y. Yamazaki, Toshiba Corp. (Japan); S. Borisov, A. Ivanchikov, S. Babin, aBeam Technologies (United States)

7272 3J Development and implementation of PWQ on patterned wafer darkfield inspection systems [7272-145]

U. Streller, K. Wendt, A. Wehner, J. Goeckeritz, Qimonda Dresden GmbH \& Co. OHG

(Germany); M. Gahr, M. Tuckermann, KLA-Tencor GmbH (Germany); J. Kopp, M. Hellerqvist, KLA-Tencor Corp. (United States)

7272 3K Development of a novel methodology for effective partial die inspection and monitoring [7272-147]

B.-H. Lee, T.-Y. Lee, SAMSUNG Electronics Co., Ltd. (Korea, Republic of); A. Cross, M. Aoki,

H. Choi, Y. Pae, KLA-Tencor Corp. (United States)

7272 3L Evaluation of a new photoresist dispense system to detect coating variation [7272-148] F. Gapin, B. Le-Peutrec, Altis Semiconductor (France); L. Stock, M. Hanotte, Entegris S.A.S. (France) 
$72723 \mathrm{M} \quad$ A study on effect of point-of-use filters on defect reduction for advanced 193nm processes [7272-149]

N. Vitorino, E. Wolfer, Y. Cao, D. Lee, AZ Electronic Materials USA Corp. (United States);

A. Wu, Entegris, Inc. (United States)

$72723 \mathrm{~N} \quad$ Automated defect review of the wafer bevel with a defect review scanning electron microscope [7272-150]

S. McGarvey, Hitachi High Technologies America, Inc. (United States); M. Kanezawa, Hitachi High-Technologies Corp (Japan)

727230 Results from prototype die-to-database reticle inspection system [7272-151]

B. Mu, A. Dayal, B. Broadbent, P. Lim, A. Goonesekera, C. Chen, K. Yeung, B. Pinto, KLA-Tencor Corp. (United States)

7272 3P Automated reticle inspection data analysis for wafer fabs [7272-152]

D. Summers, G. Chen, Freescale-ATMC (United States); B. Reese, T. Hutchinson,

M. Liesching, H. Ying, R. Dover, KLA-Tencor Corp. (United States)

$72723 Q \quad$ Inspection and metrology tools benefit from free-form refractive micro-lens and micro-lens arrays [7272-153]

T. Bizjak, T. Mitra, L. Aschke, LIMO Lissotschenko Mikrooptik GmbH (Germany)

$72723 R \quad$ A scatterometry-based CD metrology solution for advanced nodes, including capability of handling birefringent layers with uniaxial anisotropy [7272-155]

C.-M. Ke, J. Hu, W. Wang, J. Huang, H. L. Chung, C. R. Liang, V. Shih, H. H. Liu, H. J. Lee, J. Lin, Y. D. Fan, T. Yen, Taiwan Semiconductor Manufacturing Co. Ltd. (Taiwan); N. Wright,

R. Alvarez Sanchez, W. Coene, M. Noot, K. Yuan, V. Wang, K. Bhattacharyya,

K. van der Mast, ASML Netherlands B.V. (Netherlands)

727235 An inverse ellipsometric problem for thin film characterization: comparison of different optimization methods [7272-156]

A. Akbalık, S. Soulan, J.-H. Tortai, D. Fuard, I. Kone, Lab. des Technologies de la Microelectronique, CNRS (France); J. Hazart, LETI, CEA (France); P. Schiavone, Lab. des Technologies de la Microelectronique, CNRS (France) and CNRS, Georgia Institute of Technology (United States)

7272 3T Analysis of Köhler illumination for $193 \mathbf{~ n m ~ s c a t t e r f i e l d ~ m i c r o s c o p e ~ [ 7 2 7 2 - 1 5 7 ] ~}$ Y. J. Sohn, R. Quintanilha, L. Howard, R. M. Silver, National Institute of Standards and Technology (United States)

$72723 \mathrm{U} \quad$ A new illumination technique for grating-based nanometer measurement applications [7272-158]

L. Jiang, Tuskegee Univ. (United States)

$72723 \mathrm{X}$ SCATT: software to model scatterometry using the rigorous electromagnetic theory [7272-161]

S. Babin, L. Doskolovich, aBeam Technologies (United States); Y. Ishibashi, Toshiba Corp. (Japan); A. Ivanchikov, N. Kazanskiy, I. Kadomin, aBeam Technologies (United States); T. Mikami, Y. Yamazaki, Toshiba Corp. (Japan) 
$72723 Y$ Improved diffraction computation with a hybrid C-RCWA-method [7272-162]

J. Bischoff, Timbre Technologies, Inc. (United States) and Tokyo Electron Germany GmbH (Germany)

$72723 Z$ Multi-purpose optical profiler for characterization of materials, film stacks, and for absolute topography measurement [7272-163]

X. Colonna de Lega, M. Fay, P. de Groot, B. Kamenev, J. R. Kruse, M. Haller, Zygo Corp. (United States); M. Davidson, Spectel Research Corp. (United States); L. Miloslavsky, D. Mills, Western Digital Corp. (United States)

$727240 \quad$ Immersion scanner proximity matching using angle resolving scatterometry metrology [7272-164]

R.-J. Kou, R. Jungblut, J. Hauschild, S.-E. Tseng, J. Shieh, J. Chen, A. Chen, ASML Taiwan Ltd. (Taiwan); K. Schreel, ASML Netherlands B.V. (Netherlands)

$727241 \quad$ Integrated ODP metrology with floating n\&k's for lithography process [7272-165] P. Kearney, Tokyo Electron Europe Ltd. (Germany); D. Likhachev, Timbre Technologies, Inc. (United States); J. Uchida, Tokyo Electron Europe Ltd. (Germany); G. Fleischer, Qimonda Dresden $\mathrm{GmbH} \& \mathrm{Co}$. OHG (Germany)

727243 Restoring pattern CD and cross-section using scatterometry: various approaches [7272-168] S. Babin, L. Doskolovich, E. Kadomina, I. Kadomin, S. Volotovskiy, aBeam Technologies (United States)

727244 Process optimization for optical CD correlation improvement of ADI and AEI 3D structure by using iODP [7272-169]

A. Lee, C.-Y. Lin, F. Y. Chen, W.-H. Chang, Nanya Technology Corp. (Taiwan); S. Hsu, Hermes Systems Inc. (Taiwan); A. Li, Tokyo Electron Taiwan Ltd. (Taiwan); Y. Luo, Y. Wen, Timbre Technologies, Inc. (United States)

$727245 \quad$ Using scatterometry to improve process control during the spacer pitch splitting process [7272-171]

S. Corboy, C. MacNaughton, T. Gubiotti, M. Wollenweber, KLA-Tencor Corp. (United States)

727248 Time dependence of SEM signal due to charging: measurements and simulation using Monte Carlo software [7272-174]

H. Abe, Toshiba Corp. (Japan); S. Babin, S. Borisov, aBeam Technologies (United States); A. Hamaguchi, Toshiba Corp. (Japan); A. Ivanchikov, aBeam Technologies (United States); M. Kadowaki, Y. Yamazaki, Toshiba Corp. (Japan)

727249 Arbitrary precision value overlay and alignment system by double positioning of mask and wafer and electronic datum and nano sensor [7272-175]

W. L. Bear, Wynnbear Leading Lithography Inc. (United States); X.-W. Xiong, Wynnbear International DP and EUV lithography Inc. (China)

7272 4B Noise-free estimation of spatial line edge/width roughness parameters [7272-177]

V. Constantoudis, E. Gogolides, Institute of Microelectronics, NCSR Demokritos (Greece) 
7272 4C Comparison of physical gate-CD with in-die at-speed non-contact measurements for bin-yield and process optimization [7272-178]

J. S. Vickers, tau-Metrix Inc. (United States); J. Galvier, W. Doedel, Crolles II Alliance (France); G. Steinbrueck, tau-Metrix Inc. (United States); B. Borot, M. Gatefait, P. Gouraud, P. Gros, Crolles II Alliance (France); G. Johnson, M. Babazadeh, M. Pelella, N. Pakdaman, tau-Metrix Inc. (United States)

7272 4D Implementation of multiple ROI with single FOV for advanced mask metrology [7272-179] K. Jeong, H. Frezghi, M. Tavassoli, Intel Corp. (United States); S. Kim, Synopsys, Inc. (United States); R. Morgan, SEMI North America (United States)

Author Index 
Downloaded From: https://www.spiedigitallibrary.org/conference-proceedings-of-spie on 25 Apr 2023

Terms of Use: https://www.spiedigitallibrary.org/terms-of-use 


\title{
Symposium Committees
}

\author{
Symposium Chair
}

Christopher J. Progler, Photronics, Inc. (United States)

Symposium Cochair

Donis G. Flagello, Nikon Corporation of America (United States)

Technical Organizing Committees

Executive Committee

John A. Allgair, SEMATECH Inc. (United States) and Advanced Micro Devices, Inc. (United States)

Mircea V. Dusa, ASML MaskTools, Inc. (United States)

Donis G. Flagello, Nikon Corporation of America (United States)

Clifford L. Henderson, Georgia Institute of Technology (United States)

Bruno La Fontaine, Advanced Micro Devices, Inc. (United States)

Harry J. Levinson, Advanced Micro Devices, Inc. (United States)

Christopher J. Progler, Photronics, Inc. (United States)

Christopher J. Raymond, Nanometrics, Inc. (United States)

Michael L. Rieger, Synopsys, Inc. (United States)

Franklin M. Schellenberg, Mentor Graphics Corporation (United States)

Vivek K. Singh, Intel Corporation (United States)

Advisory Committee

Robert D. Allen, IBM Almaden Research Center (United States)

William H. Arnold, ASML US Inc. (United States)

Timothy A. Brunner, IBM Thomas J. Watson Research Center (United States)

Ralph R. Dammel, AZ Electronic Materials USA Corporation (United States)

Roxann L. Engelstad, University of Wisconsin, Madison (United States)

Roderick R. Kunz, MIT Lincoln Laboratory (United States)

Harry J. Levinson, Advanced Micro Devices, Inc. (United States)

Burn Lin, Taiwan Semiconductor Manufacturing Company, Ltd. (Taiwan)

Chris A. Mack, LithoGuru.com (United States)

Victor Pol, Freescale Semiconductor, Inc. (United States) 
Michael T. Postek, National Institute of Standards and Technology (United States)

Luc Van den Hove, IMEC vzW (Belgium)

C. Grant Willson, The University of Texas at Austin (United States) Anthony Yen, Taiwan Semiconductor Manufacturing Company, Ltd. (Taiwan) 


\title{
Conference Committee
}

\author{
Conference Chair
}

John A. Allgair, SEMATECH Inc. (United States) and Advanced Micro Devices, Inc. (United States)

Conference Cochair

Christopher J. Raymond, Nanometrics Inc. (United States)

Program Committee

Ofer Adan, Applied Materials, Inc. (Israel)

Michael E. Adel, KLA-Tencor Corporation (Israel)

Chas N. Archie, IBM Microelectronics Division (United States)

Jason P. Cain, Advanced Micro Devices, Inc. (United States)

Alain G. Deleporte, STMicroelectronics (France)

Daniel J. C. Herr, Semiconductor Research Corporation (United States)

David C. Joy, The University of Tennessee (United States)

Chih-Ming Ke, Taiwan Semiconductor Manufacturing Company Ltd. (Taiwan)

Byoung-Ho Lee, SAMSUNG Electronics Company, Ltd. (South Korea)

Martha I. Sanchez, IBM Almaden Research Center (United States)

Richard M. Silver, National Institute of Standards and Technology

(United States)

Alexander Starikov, Intel Corporation (United States)

Neal T. Sullivan, Arradiance, Inc. (United States)

Vladimir A. Ukraintsev, Veeco Instruments Inc. (United States)

Session Chairs

1 Keynote Session

John A. Allgair, SEMATECH Inc. (United States) and Advanced Micro Devices, Inc. (United States)

Christopher J. Raymond, Nanometrics Inc. (United States)

2 Methods for Today

Jason P. Cain, Advanced Micro Devices, Inc. (United States)

Alexander Starikov, Intel Corporation (United States)

3 Solutions for Tomorrow

Christopher J. Raymond, Nanometrics Inc. (United States)

Daniel J. C. Herr, Semiconductor Research Corporation (United States) 
Daniel J. C. Herr, Semiconductor Research Corporation (United States)

Jason P. Cain, Advanced Micro Devices, Inc. (United States)

$5 \quad$ Line Edge/Width Roughness

Martha I. Sanchez, IBM Almaden Research Center (United States)

Vladimir A. Ukraintsev, Veeco Instruments Inc. (United States)

6 SEM I

John A. Allgair, SEMATECH Inc. (United States) and Advanced Micro Devices, Inc. (United States)

Alexander Starikov, Intel Corporation (United States)

7 Scatterometry I

Christopher J. Raymond, Nanometrics Inc. (United States)

Richard M. Silver, National Institute of Standards and Technology (United States)

8 SEM II

Ofer Adan, Applied Materials, Inc. (Israel)

John A. Allgair, SEMATECH Inc. (United States) and Advanced Micro Devices, Inc. (United States)

9 Diffraction-Based Overlay

Christopher J. Raymond, Nanometrics Inc. (United States)

Vladimir A. Ukraintsev, Veeco Instruments Inc. (United States)

10 Mask Metrology

Martha I. Sanchez, IBM Almaden Research Center (United States)

Christopher J. Raymond, Nanometrics Inc. (United States)

11 Inspection

Jason P. Cain, Advanced Micro Devices, Inc. (United States)

Alexander Starikov, Intel Corporation (United States)

12 Process Control

Martha I. Sanchez, IBM Almaden Research Center (United States)

Christopher J. Raymond, Nanometrics Inc. (United States)

13 Scatterometry II

Martha I. Sanchez, IBM Almaden Research Center (United States)

Alexander Starikov, Intel Corporation (United States)

14 Reference Metrology

Christopher J. Raymond, Nanometrics Inc. (United States)

Vladimir A. Ukraintsev, Veeco Instruments Inc. (United States) 